\title{
Covid-19: Mercedes F1 to provide breathing aid as alternative to ventilator
}

\author{
Elisabeth Mahase
}

The BMJ

Mercedes F1 will produce continuous positive airway pressure (CPAP) machines for the NHS to use on covid-19 patients with serious lung infections, as a less invasive alternative to ventilators.

In collaboration with mechanical engineers at University College London and clinicians at University College London Hospital (UCLH), the Formula One company has produced a CPAP machine that can be rapidly reproduced. It has now been approved by the Medicines and Healthcare Products Regulatory Agency.

One hundred devices are being delivered to UCLH for clinical trials, with many more planned for roll out across the country.

CPAP machines - used for sleep apnoea-work by pushing an air-oxygen mix into the mouth and nose at a continuous rate. They can work for covid-19 related pneumonia-which affects the ability of the alveoli to absorb oxygen-because the pressure allows the alveoli to stay open when the patient breathes out, supporting oxygenation and making breathing easier. In comparison, invasive ventilators deliver directly into the lungs, requiring heavy sedation and a tube placed into the patient's trachea.

CPAP can therefore be a good alternative for many patients, as it is effective while being less invasive, does not require specialist nursing or intensive monitoring, and patients can be weaned off and put back on again if needed.

These machines have been widely used for patients in Italy and China with covid-19 when oxygen alone is insufficient. Around $50 \%$ of patients in Italy given CPAP were reported as having avoided the need for mechanical ventilation. Despite this, there is a short supply of the machines in the $\mathrm{UK}$, a problem that Mercedes could solve.
UCLH critical care consultant Mervyn Singer said, "These devices will help to save lives by ensuring that ventilators, a limited resource, are used only for the most severely ill.

"While they will be tested at UCLH first, we hope they will make a real difference to hospitals across the UK by reducing demand on intensive care staff and beds, as well as helping patients recover without the need for more invasive ventilation."

In the UK concerns have been raised over a lack of ventilators, which are needed for patients who develop a severe form of the disease. The World Health Organization estimated that one in six patients with the virus will become seriously ill and develop breathing difficulties.

Rebecca Shipley, director of UCL Institute of Healthcare Engineering, said, "It's been a privilege to work closely with our clinical colleagues and with doctors leading the covid-19 response in China and Italy. This close contact has helped us to define the need and respond with technology that we hope will support the NHS in the weeks and months to come."

Previous NHS guidance for covid-19 pneumonia said CPAP should not be used, however these guidelines have now been updated to include CPAP as part of the treatment pathway.

Andy Cowell, managing director of Mercedes-AMG High Performance Powertrains, said, "We have been proud to put our resources at the service of UCL to deliver the CPAP project to the highest standards and in the fastest possible time frame."

There are concerns, however, around the use of these machines in those with contagious respiratory infections, as leaks around the mask can release droplet spray from the patients' airways to attending staff. This could be a problem if staff do not have appropriate protective gear. 